\title{
Effects of short-term piano training on measures of finger tapping, somatosensory perception and motor-related brain activity in patients with cerebral palsy
}

\author{
This article was published in the following Dove Press journal: \\ Neuropsychiatric Disease and Treatment \\ 25 October 2017 \\ Number of times this article has been viewed
}

\author{
Ana Alves-Pinto' \\ Stefan Ehrlich ${ }^{2}$ \\ Gordon Cheng ${ }^{2}$ \\ Varvara Turova' \\ Tobias Blumenstein' \\ Renée Lampe' \\ 'Research Unit of the Buhl- \\ Strohmaier Foundation for Paediatric \\ Neuroorthopaedics and Cerebral \\ Palsy, Department of Orthopaedics, \\ Klinikum Rechts der Isar, Technical \\ University of Munich, Munich, \\ Germany; ${ }^{2}$ Chair for Cognitive \\ Systems, Department of Electrical \\ and Computer Engineering, Technical \\ University of Munich, Munich, \\ Germany
}

\begin{abstract}
Playing a musical instrument demands the integration of sensory and perceptual information with motor processes in order to produce a harmonic musical piece. The diversity of brain mechanisms involved and the joyful character of playing an instrument make musical instrument training a potential vehicle for neurorehabilitation of motor skills in patients with cerebral palsy (CP). This clinical condition is characterized by motor impairments that can affect, among others, manual function, and limit severely the execution of basic daily activities. In this study, adolescents and adult patients with $\mathrm{CP}$, as well as a group of typically developing children learned to play piano for 4 consecutive weeks, having completed a total of 8 hours of training. For ten of the participants, learning was supported by a special technical system aimed at helping people with sensorimotor deficits to better discriminate fingers and orient themselves along the piano keyboard. Potential effects of piano training were assessed with tests of finger tapping at the piano and tests of perception of vibratory stimulation of fingers, and by measuring neuronal correlates of motor learning in the absence of and after piano training. Results were highly variable especially among participants with CP. Nevertheless, a significant effect of training on the ability to perceive the localization of vibrations over fingers was found. No effects of training on the performance of simple finger tapping sequences at the piano or on motor-associated brain responses were registered. Longer periods of training are likely required to produce detectable changes.
\end{abstract}

Keywords: music, neurorehabilitation, sensorimotor, integration, alpha event-related desynchronization

\section{Introduction}

In recent years, there has been an increasing interest in the use of musical instrument training in the rehabilitation of motor impairments due to neuronal damage. ${ }^{1,2}$ Playing a musical instrument demands and engages multiple skills, from motor coordination to integration of motor and sensory (eg, auditory, visual, somatosensory) information. Integrating multiple types of information and translating them into actions, as during musical instrument playing, is likely to promote sensorimotor processes and drive neuroplasticity. ${ }^{3}$ Neuroplasticity changes associated with long-term musical instrument training have been extensively investigated in studies involving both professional musicians and nonmusicians and have shown changes both at functional ${ }^{4}$ and structural levels, ${ }^{5-7}$ some of which underlie motor learning..$^{8}$ Importantly, neuroplasticity changes have been reported not only after long-term training but also in nonmusicians after
Correspondence: Renée Lampe Unit of the Buhl-Strohmaier Foundation, Department of Orthopedics, Klinikum Rechts der Isar, Technical University of Munich, Ismaninger Strasse 22, 81675 Munich, Germany Tel +498941406360

Fax +49894I404849 Email renee.lampe@tum.de 
short periods of musical instrument practice. ${ }^{9,10}$ In addition to this, plasticity can be triggered not only in healthy individuals but also in patients with motor disorders due to neurological damage, such as stroke patients, ${ }^{1,2,11}$ opening the door for the exploration of musical instrument training for rehabilitation purposes, for example, in cases of motor disorders.

Cerebral palsy (CP) is a clinical condition characterized by motor impairments resulting from injury to brain structures during early brain development, pre-, peri- or postnatal. ${ }^{12}$ Manual skills are often affected, and motor deficits are frequently accompanied by sensory impairments, learning difficulties, epilepsy and attention problems. In particular, sensory deficits can be interconnected with motor impairments. ${ }^{13-18}$ While management of orthopedic problems, spasticity and weakness remains at the front line of medical care in $\mathrm{CP}$, the evidence for brain plasticity throughout life as well as technological advances has promoted research in $\mathrm{CP}$ to explore complementary therapies. ${ }^{19}$ The complex clinical framework in CP is likely to benefit from a therapeutic approach that involves multiple stimuli and processes and promotes motor learning and sensory-motor integration, such as the musical instrument training. In addition, the entertaining and joyful character of music, together with the drive for mastering a musical instrument, constitutes additional advantages that are not to be underestimated in the context of rehabilitation of adolescents and patients requiring lifelong therapeutic training.

Previous studies of piano training by patients with $\mathrm{CP}$ have delivered encouraging results in terms of development of manual skills and neuroplasticity effects. ${ }^{20-22}$ The heterogeneity of clinical symptoms and causes of CP, together with the additional disorders that can accompany motor deficits and the general complex clinical picture of each patient, has however partially hindered the finding of experimental evidence for the benefit of piano training in the rehabilitation of sensorimotor disorders in CP.

The current study extended this previous work by investigating the effects of piano training in the rehabilitation of sensorimotor processes in adolescents with CP in the context of training the Mozart piano concert and as preparation for a group performance of that piece. The preparation for a concert was expected to increase motivation for and the efficacy of the training. Motivation for training is an important aspect in CP, since patients present often attentional deficits, in addition to motor impairment, and therapy usually continues throughout life. Training was helped in a few cases by the use of a technical system especially developed for training persons with sensorimotor deficits, to learn to play the piano. ${ }^{23}$
The study attempted to overcome some of the difficulties faced in previous studies, for instance, by using a repeatedmeasures experimental design to investigate the effects of piano training on the rehabilitation of sensorimotor processes in CP. By assessing experimental correlates of sensorimotor processes in the same individual in different experimental conditions, that is, in the absence of and after piano training, we tried to overcome the difficulty in finding matched groups of patients. A group of typically developing adolescents was also included in the study to verify whether motor learning would be possible in this short period of training in the absence of sensorimotor disorders. Rehabilitation effects were sought by measuring 1) changes in the dynamics of keystrokes while playing simple sequences at the piano, 2 ) changes in the ability to perceive and localize vibration stimuli delivered to the fingers and 3 ) changes in neuronal activity associated with motor learning. ${ }^{24-26}$

Participants with different types of CP were included in the study to explore the potential effects of piano training across different forms of the condition. The Mozart piano concert was adapted by a professional musician to the motor abilities of each participant in the study so that all could participate in the concert.

\section{Methods}

\section{Participants}

The test group was composed of nine adolescents with a diagnosis of CP (named CPY group; 11-17 years, mean age of 15 years; Table 1). A group of six healthy, typically developing adolescents participated as a control group (named CTR group; 7-17 years, mean age 12 years). A third group of seven adults with CP (named CPA group; 34-52 years, mean age of 44 years) was also included to explore effects of piano training. The level of motor impairment, according to the Gross Motor Function Classification System, ${ }^{12}$ varied from 1 to 4 , with two participants having the maximum level of impairment. The degree of manual skill, described according to the Manual Ability Classification System, ${ }^{27}$ varied between I and $\mathrm{V}$.

Adult participants with $\mathrm{CP}$ were recruited from a center for disabled persons with assisted living in Munich. All adolescents with CP attend an integrative school center in Munich for children with physical disability. Adolescents typically spend their day at the center, where they can attend not only school classes but also therapeutic training and freetime activities, being accompanied by care professionals all the time. Control participants were relatives of members of the team and of the personnel working at the care and school centers mentioned above, all also living in Munich. 
Table I Demographic and clinical data of all study participants: control group (CTR), adults with cerebral palsy (CPA) and adolescents with cerebral palsy (CPY)

\begin{tabular}{|c|c|c|c|c|c|c|c|c|c|c|}
\hline Participant & Group & Gender & $\begin{array}{l}\text { Age, } \\
\text { years }\end{array}$ & $\begin{array}{l}\text { Best } \\
\text { hand }\end{array}$ & GMFCS & MACS L & MACS R & Diagnose & $\begin{array}{l}\text { Support } \\
\text { system }\end{array}$ & $\begin{array}{l}\text { Hand } \\
\text { tested }\end{array}$ \\
\hline sll & CPA & $\mathrm{F}$ & 44 & $R$ & 3 & II & II & Bilateral leg-affected spastic CP & & Both \\
\hline $\mathrm{s} 12$ & CPA & M & 37 & L & 3 & III & III & Bilateral spastic CP & & Both \\
\hline$s \mid 3$ & CPA & $\mathrm{F}$ & 45 & L & 2 & II & $\mathrm{V}$ & Unilateral spastic CP & & L \\
\hline sl4 & CPA & M & 48 & L & 3 & II & II & Bilateral R-affected CP & y & Both \\
\hline sl5 & CPA & $\mathrm{F}$ & 52 & $\mathrm{R}$ & 4 & II & II & Ataxic $\mathrm{CP}$ & y & Both \\
\hline s16 & CPA & $\mathrm{F}$ & 50 & L & 3 & ॥ & III & Bilateral R-affected CP + distonia & $y$ & Both \\
\hline s17 & CPA & M & 34 & $\mathrm{R}$ & 4 & IV & IV & Bilateral spastic CP & & $\mathrm{R}$ \\
\hline s30 & CPY & M & 15 & $\mathrm{R}$ & 3 & III & $\|$ & Dyskinetic CP & & $\mathrm{R}$ \\
\hline s31 & CPY & $\mathrm{F}$ & 17 & $\mathrm{R}$ & I & I & $\mathrm{I}$ & Ataxic $\mathrm{CP}$ & y & Both \\
\hline s32 & CPY & M & 15 & L & I & I & II & Acquired unilateral $\mathrm{CP}$ & & Both \\
\hline s33 & CPY & M & 17 & L & 2 & II & II & Bilateral spastic CP & y & Both \\
\hline s34 & CPY & $\mathrm{F}$ & 16 & $\mathrm{R}$ & 2 & II & II & Arthrogryposis multiplex congenita & & Both \\
\hline s35 & CPY & $\mathrm{F}$ & 14 & $\mathrm{R}$ & 3 & III & II & Bilateral CP & y & Both \\
\hline s36 & CPY & $\mathrm{F}$ & II & L & 2 & II & III & Unilateral spastic CP & & Both \\
\hline s37 & CPY & M & 15 & $\mathrm{R}$ & 3 & III & II & Bilateral spastic CP & y & $\mathrm{R}$ \\
\hline s38 & CPY & $\mathrm{F}$ & 15 & $\mathrm{R}$ & I & 1 & I & Ataxic $\mathrm{CP}$ & & Both \\
\hline s20 & CTR & $\mathrm{F}$ & 14 & $\mathrm{R}$ & & & & & & Both \\
\hline s2I & CTR & $\mathrm{F}$ & 7 & $R$ & & & & & $y$ & Both \\
\hline$s 22$ & CTR & $\mathrm{F}$ & II & L & & & & & $y$ & Both \\
\hline$s 23$ & CTR & $M$ & 17 & $R$ & & & & & $y$ & Both \\
\hline s24 & CTR & $M$ & 11 & $R$ & & & & & & Both \\
\hline s25 & CTR & $M$ & 13 & $\mathrm{R}$ & & & & & & \\
\hline
\end{tabular}

Abbreviations: GMFCS, Gross Motor Function Classification System; MACS, Manual Ability Classification System; L, left hand; R, right hand; F, female; M, male; CP, cerebral palsy; y, yes.

Researchers, teachers and/or caregivers approached potential participants (adults and adolescents with $\mathrm{CP}$ ) and inquired about their interest in participating in the study. The analysis here presented is based on data collected for all participants who showed an interest and accepted to participate in the study (there were no drop-offs). All participants and parents or legal guardians, in case of adolescents or adults with CP with a legal guardian, were informed about the aims and procedures of the study through personal communication and in written form before the beginning of the study. Written consent was provided by adult participants, by the parents of under-legal-age participants and, in a few cases, by the patients' legal guardians on behalf of the participants before the beginning of the experimental tests. All experimental procedures were approved by the Ethics Committee of the Medical Faculty of the Technical University of Munich before the beginning of the study.

\section{Experimental design and procedure}

Experimental tests were conducted three times, with four weeks separating each session (Figure 1A). Piano training took place in the second 4-week period, between the second and the third experimental session. This design with three sessions was originally intended to distinguish changes in experimental variables due to piano training from changes occurring as part of normal physical/manual cognitive development in young participants. This experimental design avoided furthermore the need for having matched groups of patients with $\mathrm{CP}$ who would receive and would not receive training, particularly difficult given the heterogeneity of causes and symptoms across this clinical population.

\section{Piano training}

Participants received individual training with a professional piano teacher twice a week for 4 weeks, making a total of 8 hours of training per person. For participants with CP, training took place at the care center or at the integrative school center. This was especially important for those participants with less mobility. Control participants received training at home.

Training was for all participants individualized. Prior to the study, teachers received a brief preparation about $\mathrm{CP}$, about the experience collected from previous piano studies $^{20,21}$ with these patients and about how to teach with the technical support system developed. The attention and care put by each teacher into the individual classes became reflected in the trust and in the engagement participants developed during the study.

Hand motor abilities were analyzed individually before the beginning of the study. The original piano concert was 


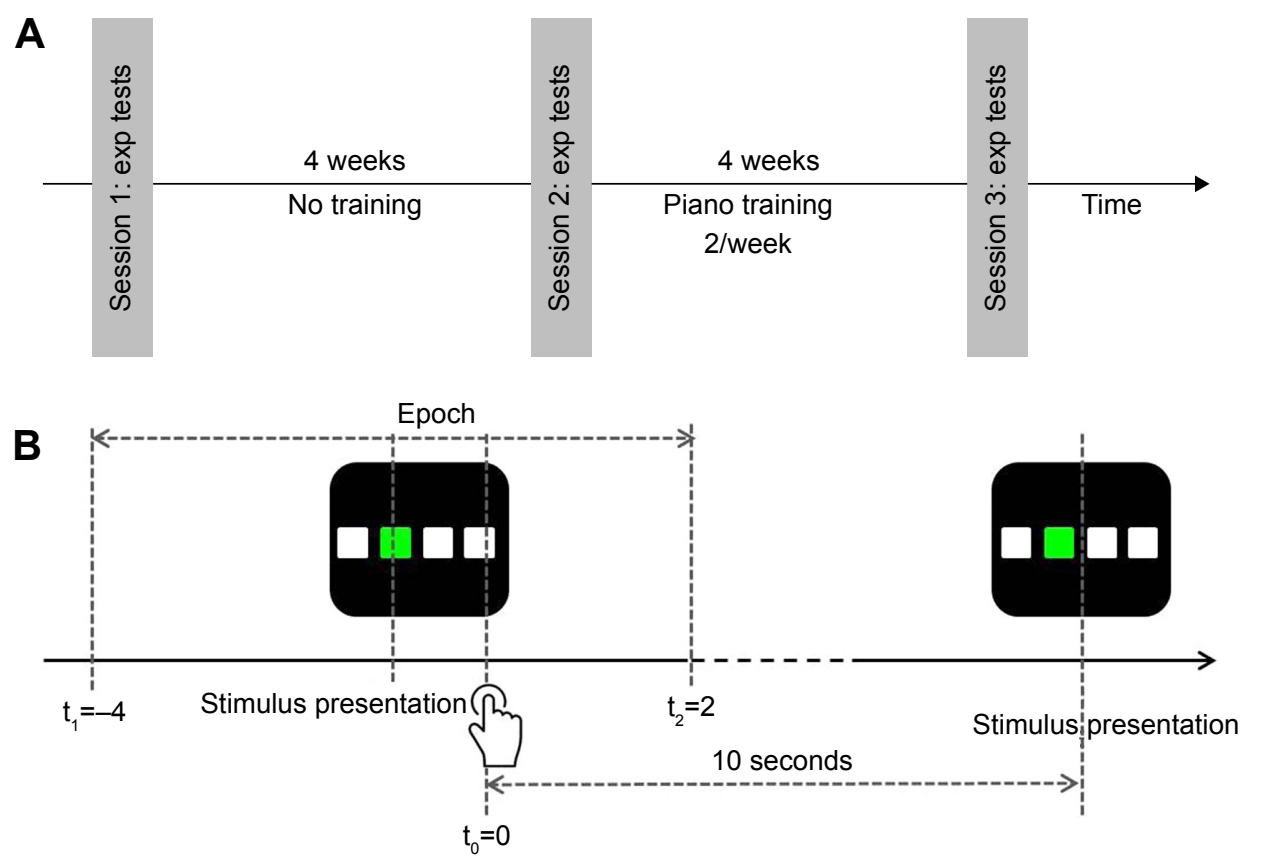

Figure I (A) Experimental design of the study. Three experimental sessions were conducted, separated by 4 weeks. Participants received piano lessons only during the 4 weeks between the second and the third experimental sessions. (B) Diagram of the experimental test used to assess alpha ERD. A trial was initiated by the presentation of four squares (three white, one green) in a screen. Participants had to indicate the location of the green square, by pressing the corresponding button in a keyboard with four buttons. Brain activity was recorded from electrodes located over and surrounding motor areas. ERD was computed in time windows starting 4 seconds before the button press ( $\mathrm{t}=-4$ seconds) and finishing 2 seconds ( $\mathrm{t}=2$ seconds) after the button press. An interval of 10 seconds separated button press and the presentation of the following stimulus.

Abbreviations: ERD, event-related desynchronization; exp, experimental.

then adapted to the manual abilities of each participant. There were eight voices in total that were distributed among the participants, patients and controls.

Teachers sat together with their pupils at the same piano keyboard. Training started by touching the piano keyboard, playing simple sequences and exploring improvization. After this, training focused on learning the part of the adapted Mozart piano concert that had been prepared for the pupil. Often during this part, teachers played together with the pupil in order to create the "musical context" of the concert, in which the piece that has been learned was inserted. Other times, teacher and pupil would also hear the concert from a music CD containing an enhanced version of the voice/part of the concert to be trained so that the pupil could learn how his/her piece fitted in the whole concert.

For ten of the participants (controls and patients), training was supported by a technical system specially developed to assist patients with sensorimotor deficits. ${ }^{23}$ The selection of patients who would train with the system was based on a clinical evaluation by an experienced medical doctor who indicated those patients showing deficits in spatial orientation and/or deficits in somatosensory perception. Control participants were selected to train with the system and were of similar age to the young patients trained with the system.
The system is composed by a bar with LEDs, a set of gloves for the pupil and a set of gloves for the teacher. The bar with LEDs is placed over the piano keyboard and is connected to the piano used by the teacher (the same or other). As the teacher plays, the LEDs corresponding to the keyboard keys struck by the teacher are lit, this way indicating the pupil which keys to press. The teacher can wear special gloves containing sensors that capture the sequence of fingers used to play that sequence. This information is transferred to the gloves of the pupil through vibrations and by lighting small LEDs over the corresponding fingers. Both LEDs and vibrations can support the learning of finger sequences, especially in cases of patients with sensorimotor disorders, who often have difficulty in discriminating between fingers. For some participants, only vibratory stimulation or only visual (LED) stimulation was employed, depending on whether the pupil reported to be of no help what the teacher felt was the best combination for the participant.

\section{Experimental tests}

Three different experimental tests were carried out: 1) hand motor tests at the piano (named "piano tests"), used to assess behaviorally manual skills by capturing changes in finger mobility with the training (if any changes were to 
occur, then they should be larger and easier to detect for measures of motor function related to the type of movements that were trained); 2) test of perception of vibration at the fingers (named "vibration tests"); and 3) measurement of brain alpha-power event-related desynchronization (ERD; named "alpha ERD") to assess potential neuronal correlates of motor learning. ${ }^{24}$ All tests were performed in a normal office room.

\section{Piano tests}

Three different tests were done at the piano:

1) Test 1: Playing 16 consecutive times the same note with the same finger, from thumb to small finger, once with right hand, once with left hand.

2) Test 2: Playing five different notes consecutively, one with each finger from thumb to small finger and in the inverse direction. Only the white keys of the piano were struck. The test was repeated three times with each hand.

3) Test 3: As in 2) but with the two hands at the same time. This test requires additional coordination between the two hands. Eight of the 16 participants with $\mathrm{CP}$ were not able to perform this test, five were unable to coordinate movements and three with unilateral $\mathrm{CP}$ were unable to produce any movements with one of the hands. Despite the latter, they were not excluded from the study since mobility of the least-affected hand could still benefit from piano training, and this would still be meaningful for the study.

Part of the piano data collected in the third experimental session was lost by an unfortunate mistake. Therefore, there are data missing for five adolescents with $\mathrm{CP}$ for the third session.

\section{Vibration tests}

A response box with five large buttons arranged in a semicircle was placed over a table in front of the participant. Five vibration motors connected to the response box were placed around each of the fingers with the help of a Velcro strip. The vibration motors were covered with a piece of tissue so that the participant could not visually identify which finger was being stimulated. A harmless $200-\mathrm{Hz}$ vibration was delivered to a finger for 1 second on each trial. The participant's task was to indicate which finger was stimulated by pressing one of five buttons, with each button corresponding to a different finger. The two hands were tested separately. Vibrations were presented randomly across fingers, with a total of ten trials presented per finger.

\section{Measurement of alpha ERD}

Participants were seated in front of a computer screen. The experimental procedure was similar to a reaction time task: on each trial, four squares were displayed horizontally in the middle of the screen (Figure 1B). Three were white, and one was green. The task consisted in pressing as fast as possible on a keyboard with four keys only, the key corresponding to the position of the green square (from 1 to 4 ). The squares disappeared from the screen immediately after the response, and a new trial was initiated with the appearance of a new set of four squares 10 seconds after the response. Forty trials were presented for each hand in each experimental session, whereby the order of presentation of stimuli was pseudorandomized. In four cases, only one hand could be tested, and in one case, the test was performed with only two fingers due to strong spasticity (Table 1, "hand tested").

Brain activity over the sensorimotor areas was recorded during the whole duration of the test. Recordings were performed with a headset specially adapted for the test population from an Emotiv EPOC ${ }^{\circledR}$ device. ${ }^{28}$ Electrodes were hidden within a structure similar to a headphone such that when the headset was in place, electrodes covered the sensorimotor area. The headset was very easy to set up and had an unobtrusive look, two important features when working with young patients with $\mathrm{CP}$, who have a long history of medical examinations and who are often reluctant in taking part in studies involving such medical tests.

Data were collected with 13 electrodes covering the following positions of the 10-20 system: FC3, FC4, C5, C3, C1, $\mathrm{Cz}, \mathrm{C} 2, \mathrm{C} 4, \mathrm{C} 6, \mathrm{CP} 3$ and CP4. Reference was the average of the signal recorded from the two mastoids (TP9 and TP10). Data collection at a sampling rate of $128 \mathrm{~Hz}$ was initiated after assuring good conductance. This was controlled by the Emotiv Testbench software and corresponded to an impedance $<220 \mathrm{k} \Omega{ }^{29}$

\section{Data analysis \\ Piano tests}

The following variables were computed from the tests performed with one hand only for each participant: 1) mean and standard deviation of the duration of keystrokes, across fingers; 2) mean and standard deviation of the time interval between consecutive keypresses; and 3) mean and standard deviation of the keystroke pressure. Given the wide variability in manual ability, and therefore in speed of finger movement, across participants, the coefficient of variation (standard deviation/mean) was used to evaluate the data. Lower coefficients of variation indicate lower 
relative variability and therefore a more regular pattern of keypresses.

For the test executed with both hands simultaneously, the level of coordination between the two hands was assessed through the nonparametric Kendall correlation coefficient computed between the patterns of keyboard presses of the left and right hands. Correlation does not consider the timing of keypresses; the sequence of fingers used to play is analyzed. A perfect coordination between the two hands, with fingers of one hand mirroring the movements of fingers of the other hand, yields a correlation coefficient of -1 . A coefficient of +1 indicates that movement occurred in the same direction in both hands (no mirror movement), for example, left small finger together with right thumb followed by left ring finger together with right pointing finger, etc.

\section{Vibration tests}

The proportion of correct responses for each hand was computed as the ratio of all correct responses, including all fingers, to the total number of 50 trials.

\section{Alpha ERD}

Data preprocessing was performed with the freely available EEGLAB toolbox, ${ }^{30}$ and data analysis was performed in MATLAB R2013b ${ }^{\circledR}$. Preprocessing included baseline correction, followed by re-referencing to $\mathrm{Cz}$ and then bandpass filtering within $[2,40] \mathrm{Hz}$ using a Hamming-windowed sinc finite impulse response (FIR) filter. Epochs were then extracted from the continuously recorded signal by selecting the time period $[-4,2]$ seconds relative to the moment of button press ( $\mathrm{t}_{0}$ in Figure 1B). This way, recorded epochs from participants with very different response times could be aligned according to a common time point - the moment of button press. Epochs with signal amplitudes larger than $\pm 40 \mu \mathrm{V}$ in the channels C3 and C4 were excluded from further analysis. These channels are the closest locations to motor areas and those for which the signal was most stable throughout recordings. Some of the other channels presented often large amplitudes and unstable signals and were excluded therefore from further processing. After this automatic selection, all epochs were visually inspected by a researcher, and epochs that presented clear artifacts (eg, from muscular movement) were excluded from analysis. For a few participants with $\mathrm{CP}$, epoch selection resulted in the exclusion of the entire experimental session.

ERD was computed, for each participant, from the set of selected epochs of the alpha-filtered signals collected from channels $\mathrm{C} 3$ and $\mathrm{C} 4$. Alpha-filtering was applied to the continuous signal with a Hamming-windowed FIR filter into $[8,13] \mathrm{Hz}$. ERD was computed on an individual basis using the intertrial variance method as described by Kalcher and Pfurtscheller ${ }^{31}$ and using the time window [-4, -3] seconds (or $[-3,-2]$ seconds for a minority of participants) before button press ( $\mathrm{t}=0$ second $)$ as a reference period.

\section{Statistical analysis}

All statistical analyses presented were done with the statistics and machine learning toolboxes of MATLAB $2015 b^{\circledR}$.

\section{Results}

\section{Piano tests}

The upper panels of Figure 2 illustrate how the coefficient of variation (standard deviation/mean) of time intervals between consecutive keypresses (piano test 1) changed with experimental session ("1", " 2 " and " 3 ") for each participant. Each symbol/value was computed from all keypresses recorded for one hand (ie, without distinguishing between fingers). Circles illustrate results of control participants, triangles of adolescents with $\mathrm{CP}$ and stars of adult patients with $\mathrm{CP}$. A lower coefficient of variation indicates regularity during keypress, while a large coefficient is indicative of a variable pattern of keypress. It can be observed that adolescents with CP present generally a less regular pattern of keypresses than their age-matched control peers. Also, adults with $\mathrm{CP}$ present a more variable pattern of keypresses. The change in performance across experimental session was evaluated by subtracting the coefficient of variation, on an individual level, between sessions 1 and 2 (no training received) and then between sessions 2 and 3 (ie, after piano training). The median values derived from the differences computed individually are plotted in the lower panels of Figure 2 for tests performed with the best (left panel) and second (right panel) hands, respectively. The group of adolescents with CP (triangles) show a large reduction (negative difference) in the coefficient of variation from the first to the second session, indicating a more regular pattern of keypresses in the second relative to the first session, that is, before the start of the piano training. Between the second and the third session, after piano training, there was a small tendency to perform less regularly (positive difference in coefficient of variation for the best hand) or no change (zero median difference for the second hand). The control group (gray circles in Figure 2) showed comparatively more stable performance with very small changes from session to session (median difference around zero). Adult patients with CP (star symbols) also showed larger changes in performance from session to session. 

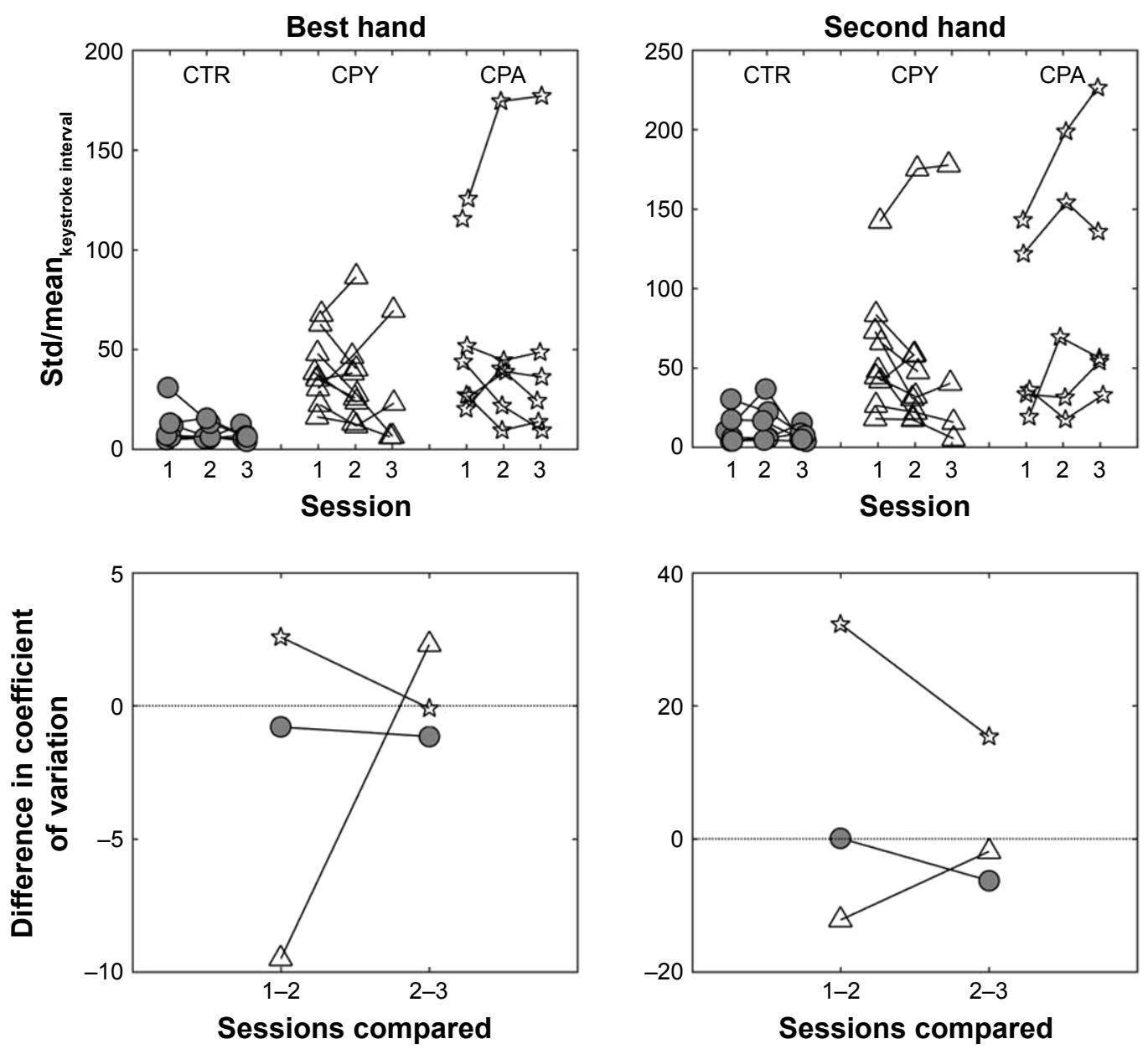

Figure 2 Upper panels: Coefficient of variation (standard deviation/mean) of time between consecutive keypresses computed from the piano test I in first, second and third experimental sessions. Each symbol corresponds to a different participant. Circles correspond to controls (CTR), triangles to adolescents with CP (CPY) and stars to adults with CP (CPA). Lower coefficients of variation indicate more regular patterns of keystrokes. Lower panels: Difference in coefficient of variation between the first and second session ("I-2" in horizontal axis) and between the second and third sessions ("2-3" in x-axis). Symbols represent the median difference across group participants. A negative difference value indicates a reduction in coefficient of variation. Left panels illustrate results obtained with the best hand, and right panels illustrate results obtained with the second hand.

Abbreviations: CP, cerebral palsy; std, standard deviation.

The lack of effect of training observed in these results was confirmed with a mixed factorial repeated-measures ANOVA on the coefficient of variation, with session (three levels: "1", "2" and "3") and hand (two levels: best and second hands) as within-subject factors, and group (three levels: "CTR", "CPY" and "CPA") and use of system (two levels: "training with system" and "training without system") as between-subject factors. No significant effects of session $(F(2,22)=0.35, p=0.71)$, or of hand $(F(1,11)=2.89$, $p=0.12)$ or of system $(F(1,11)=0.49, p=0.49)$ were found. But there was a marginally significant effect of group $(F(2,11)=3.42, p=0.07)$ as clearly observed in the upper panels of Figure 2.

No changes were observed in terms of duration of keypress or in terms of pressure at the onset of keypress, also with piano test 2 .
Playing a simple sequence with the two hands simultaneously was used to test the ability to coordinate movements with the two hands. Examples from two participants, one from the control group and an adult participant with $\mathrm{CP}$, are illustrated in Figure 3, for each of the three experimental sessions. Sequence patterns recorded with the control participant show that the movement with one hand (eg, gray lines and symbols) mirrors almost perfectly the movement of the opposite hand. This coordinated pattern remains the same from session 1 to session 2 and over to session 3 . The patterns recorded for the patient with CP (right panels) show comparatively less coordination. This patient shows in addition slight improvements for sessions 2 and 3 in comparison to the first session.

The ability to play equivalent keys with the two hands simultaneously was quantified by means of the nonparametric 

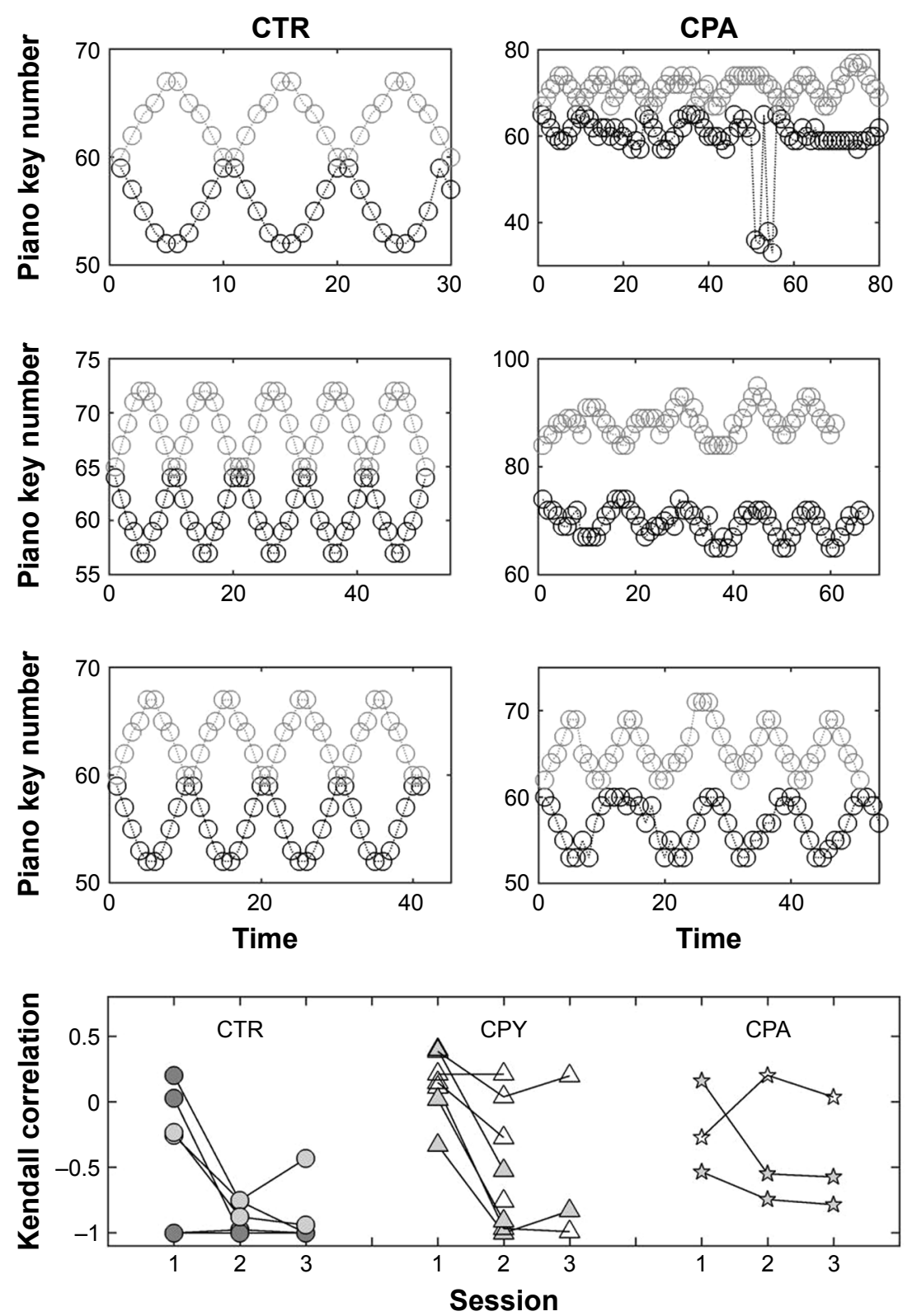

Figure 3 Three upper rows: Pattern of succession of keys struck during piano test 3 (both hands playing simultaneously) recorded from a control participant (left panels), and from an adult with CP (right panels). Lighter and darker gray symbols represent the two different hands. Upper, middle and lower panels illustrate pattern recorded in first, second and third session. Lower panel: Nonparametric Kendall correlation coefficient between the two successions of piano keys recorded with left and right hands when performing the piano test 3. Each line corresponds to the result obtained for a single participant in the three sessions. Circles correspond to controls (CTR), triangles to adolescents with $\mathrm{CP}(\mathrm{CPY})$ and stars to adults with $\mathrm{CP}(\mathrm{CPA})$.

Abbreviation: $\mathrm{CP}$, cerebral palsy.

Kendall's correlation coefficient. Results are presented in the lowest panel of Figure 3, for participants in the control group (gray circles), adolescents with CP (triangles) and adults with CP (stars). Perfect coordination corresponds to a correlation coefficient of -1 . The results show again a larger variability across adolescents and adults with $\mathrm{CP}$, independent of session. Several participants showed better coordination in the second than in the first session. Between the second and the third sessions, there was no change or if any very small.
Upper panels in Figure 4 illustrate the coefficient of variation during this piano test. Each symbol represents the coefficient of variation computed for each hand separately and for each individual, with circles representing controls, triangles adolescents with $\mathrm{CP}$ and stars adults with $\mathrm{CP}$. Differences in the coefficient of variation, computed individually, between the first and second session and between the second and the third are plotted in the lower panels. Also here, participants in all groups performed very differently. 

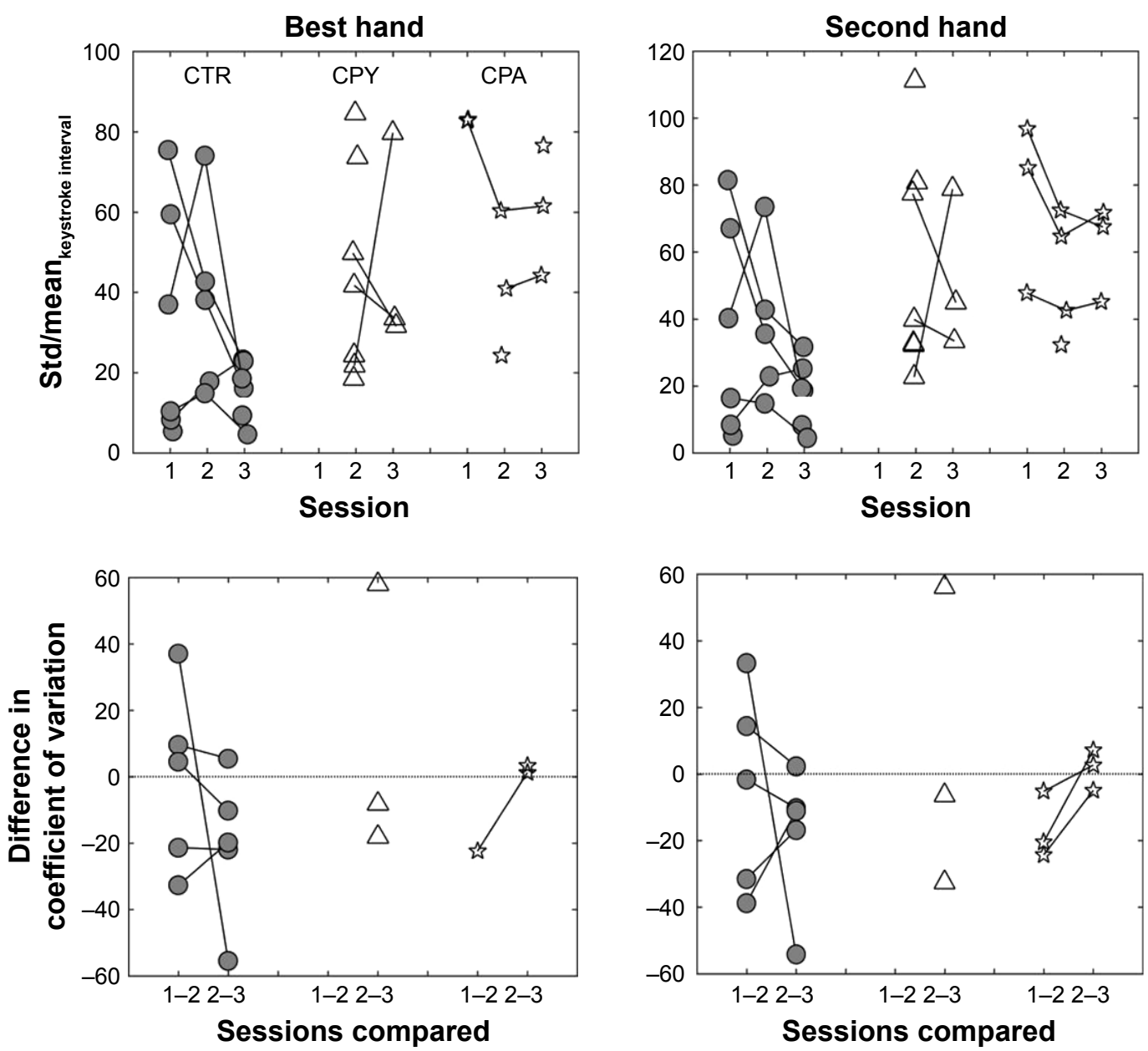

Figure 4 Upper panels: Coefficient of variation (standard deviation/mean) of time between consecutive keypresses computed from the piano test 3 in first, second and third experimental sessions, and for the best (left panels) and second hand (right panel). Circles correspond to controls (CTR), triangles to adolescents with CP (CPY) and stars to adults with CP (CPA). Lower panels: Difference in coefficient of variation between first and second session ("I-2" in horizontal axis) and between second and third session ("2-3" in X-axis). Symbols correspond to individual participants.

Abbreviation: $\mathrm{CP}$, cerebral palsy.

The coefficient of variation decreased between the first and second session (lowest panel in Figure 4) for some participants and between the second and third session for a few. Due to the lack of data for several participants, no statistical analysis was performed.

\section{Vibration tests}

The percentage of correct responses in the vibration tests is illustrated in Figure 5, for each group. Gray lines and symbols illustrate individual results, whilst black thick lines indicate mean values. A tendency for improvement in localizing vibrations can be observed for all groups from the first to the second sessions and from the second to the third sessions (after the piano training), both with the best and second hands. A mixed factorial ANOVA on percentage of correct responses (dependent variable), with group (three levels: "CTR", "CPY" and "CPA") and system (two levels: "yes" and "no") as between-subject factors, and session (three levels: "1", "2" and "3") and hand (two levels: "best" and "second") as within-subject factors, confirmed a significant effect of session $(F(2,32)=12.23, p=0.0001)$. Contrasts revealed a significant increase in the percentage of correct responses between the second and third sessions $(F(1$, 16) $=10.63, r=0.63)$, but no significant increase between the first and second sessions $(F(1,16)=1.52, r=0.29)$. There was also a significant effect of group $(F(2,16)=3.94, p=0.04)$, but no effect of system $(F(1,16)=0.002, p=0.97)$ nor of hand $(F(1,16)=2.65, p=0.12)$.

The potential association between perceptual deficits and motor impairment was sought by means of the correlation between the performance in the vibration test and the regularity of keystrokes in the piano test 1 measured in experimental session 2 (Figure 6), computed over all participants, including adults with CP. The association can 


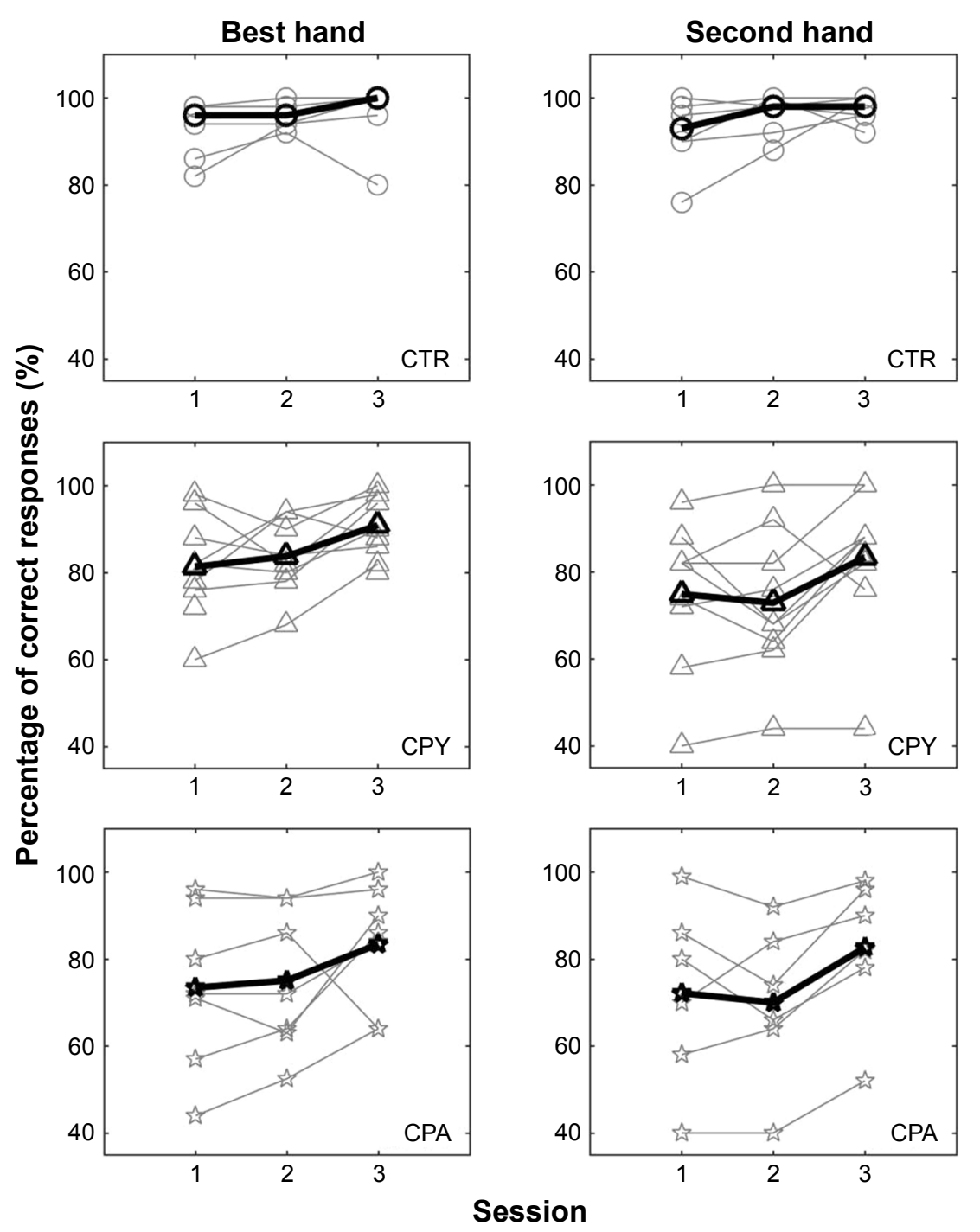

Figure 5 Percentage of correct responses obtained in the vibration test for controls (CTR; upper panels), adolescents with CP (CPY; middle panels) and adults with CP (CPA; lower panels). Percentage of correct values corresponds to the proportion of correct vibration-to-finger localizations over the total 50 trials for each hand (I0 trials per finger). Left/right panels illustrate results obtained with the best/second hand, respectively. Thin gray lines illustrate individual results. Thick black lines represent the mean percentage correct across participants.

Abbreviation: $\mathrm{CP}$, cerebral palsy.

be visualized in Figure 6 and was supported by significant correlation coefficients, for both the best $(r=-0.6, p=0.01$ ) and second $(r=-0.6, p=0.006)$ hands, respectively.

\section{Alpha ERD}

Figure 7A and B illustrates weighted ERD of alpha activity within a time frame close to button press, starting 4 seconds before the button press to 2 seconds after the button press, for three participants, one control participant (black line), one adolescent with CP (blue line) and one adult with CP (red line). All three participants show a clear reduction in alpha activity, starting after the button press for two of the participants and before the button press for the adult patient. In order to quantify the degree of ERD around response time, the average ERD in the time window $[0.5,1.5]$ seconds after the response was computed for each participant and for all three sessions. Results are plotted for the different groups in the three lower rows of Figure $7 \mathrm{C}-\mathrm{H}$. They confirm the variability in ERD across participants, with some showing only small changes and others showing larger reductions in ERD. Variability can also be observed within participants from session to session. Given the small size of the groups, that groups have different sizes and that for some participants no valid data could be collected, the nonparametric Friedman test was used to evaluate, for each group separately, the effect of session on mean ERD. No significant effect of session was obtained for the group of adolescents with CP (chi-square $(2)=0.04, p=0.98)$ nor for the control group 


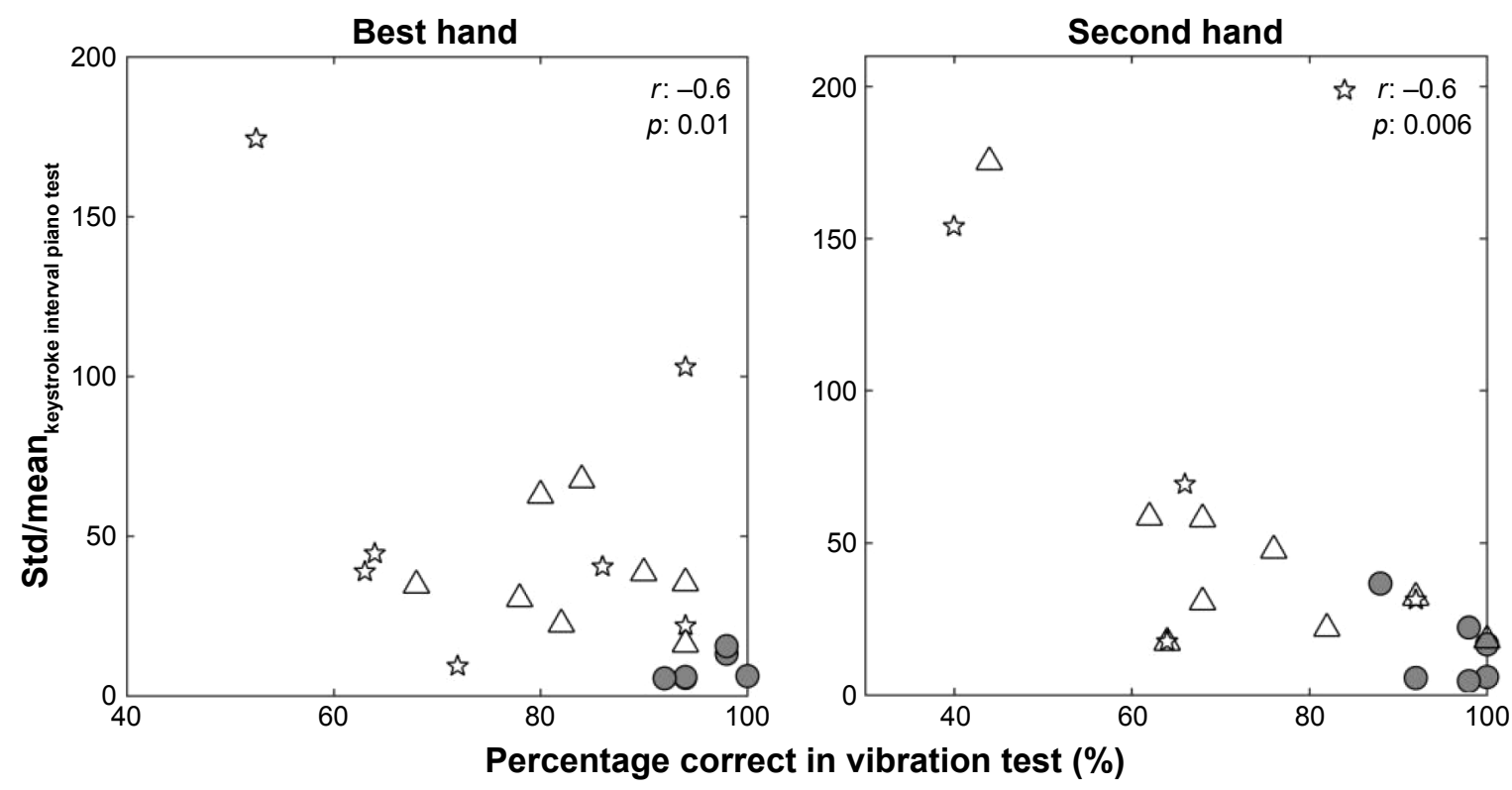

Figure 6 Coefficient of variation of the intervals between consecutive keystrokes while performing piano test I as a function of percentage of correct responses obtained in the vibration test, in session 2. Circles, triangles and stars illustrate results of individual controls, adolescents with $\mathrm{CP}$ and adults with $\mathrm{CP}$, respectively. Results obtained with the best/second hand are presented in the left/right panels, respectively. The Pearson correlation coefficient between the two variables and the associated $p$-value are also indicated.

Abbreviation: $\mathrm{CP}$, cerebral palsy.

(chi-square $(2)=2.68, p=0.26)$ nor for the group of adults with CP (chi-square (2) $=0.31, p=0.86$ ). For most participants though, mean ERD was negative, indicating that alpha power within this time window was on average lower than in the reference period before trial onset.

\section{Discussion}

An effect of training/session was obtained on tests evaluating the ability to localize vibrations delivered to fingers, with statistical contrasts showing a significant change in performance from the second to the third session, that is, after piano training. This improvement was more clearly observed for the test group of adolescents with CP and adults with CP. For controls, there was no room for improvement - localization ability was practically maximal from the beginning. Even though not all participants trained with support of the technical system, that supports learning by transmitting vibrations to fingers and indicating visually which keys to play, practicing to play the piano is likely to have promoted a more conscious awareness of the own hands and fingers than most patients are used to experience.

No significant changes in the regularity of keystrokes or in alpha ERD were found, for any of the groups tested. The lack of improvements in piano tests might indicate that a longer period of training (ie, longer than 4 weeks with 2 hours of training per week) may be necessary to operate any detectable changes in finger movement performance or in alpha ERD. The fact that no significant changes were observed for the control group, which would be expected to develop faster, as well as that the use of system did not induce a significant effect suggests that the period of training was too short. Another possible explanation is the inadequacy and/or low sensitivity of the test to potential changes in motor performance. Experimental tests were developed and selected with the objective of minimizing the testing time and also to somehow be similar to the motor skills that were being learned. Assessment of ERD was furthermore aimed at exploring a potential neuronal correlate of motor learning in rehabilitation studies. None of the tests used is a standardized test, validated by previous research. The use of a standardized test, such as the Assisting Hand Assessment, the Jebsen Taylor Test of Hand Function or the Assessment of Motor and Processing Skills, might have been more sensitive to changes in motor performance.

An additional contribution to the lack of significant results may have come from the diversity of subtypes of $\mathrm{CP}$ considered in the study. This is likely to have increased variability across patients and diminished the size of effects of training. Extending the study to different subtypes of $\mathrm{CP}$ was on the other hand intended to increase the probability of finding an effect.

In addition to these factors, results were variable across participants and sessions, especially among adolescents and adults with CP. This reduced the possibility to capture 
A
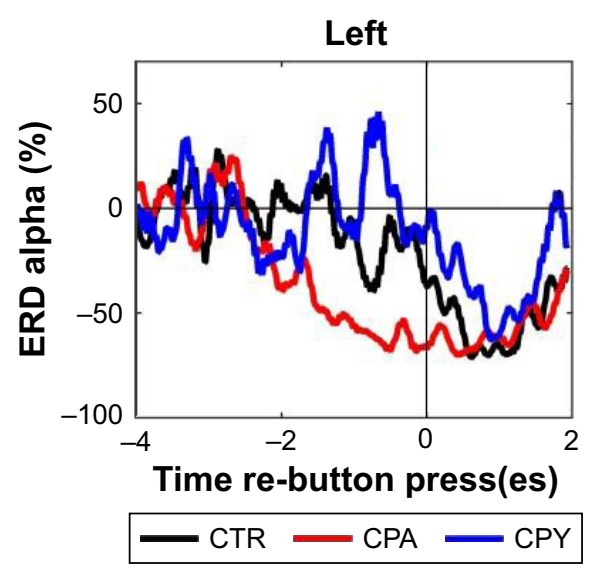

C

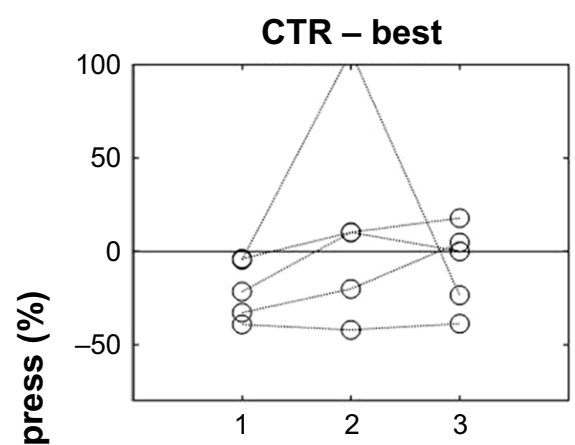

E

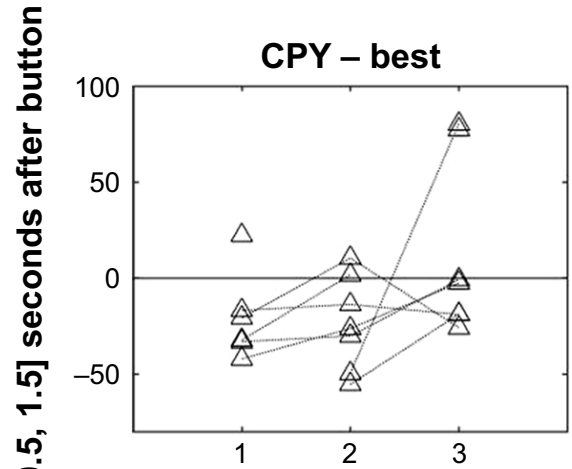

G

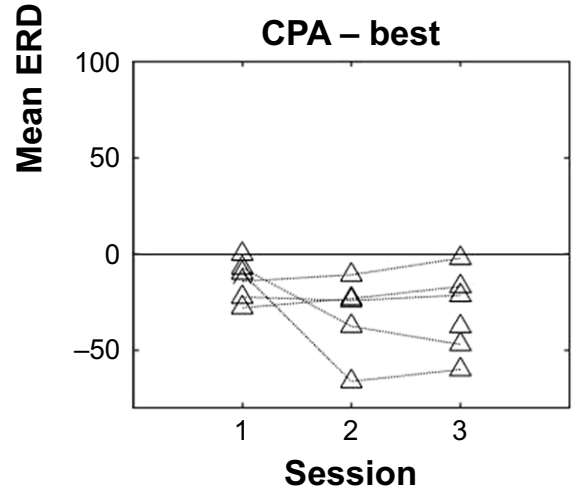

B

Right

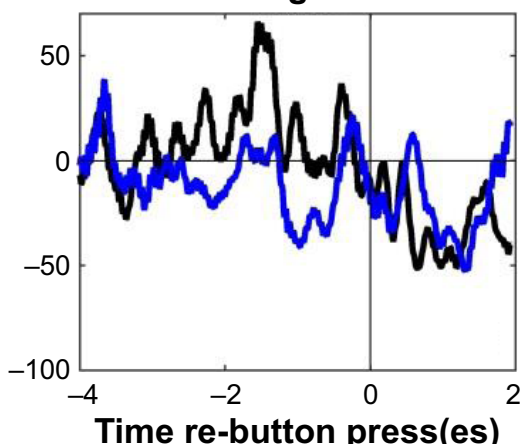

Time re-button press(es)

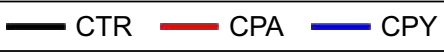

D

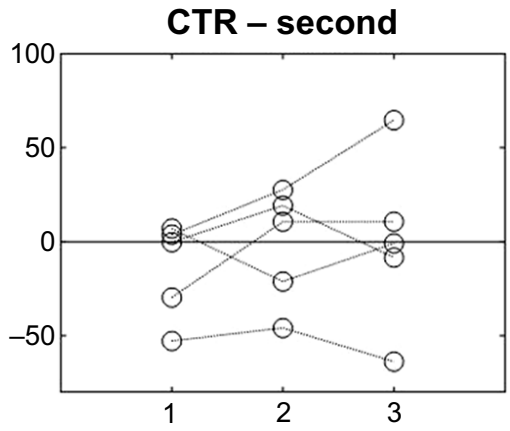

F

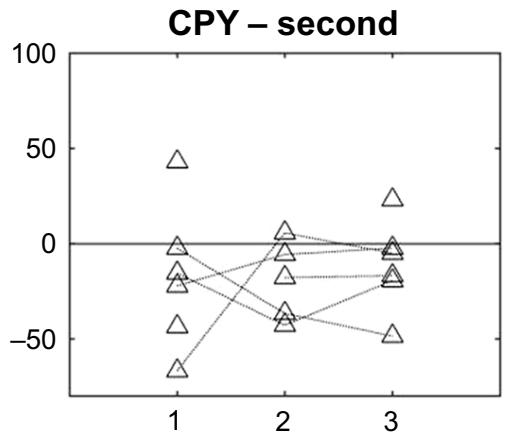

H

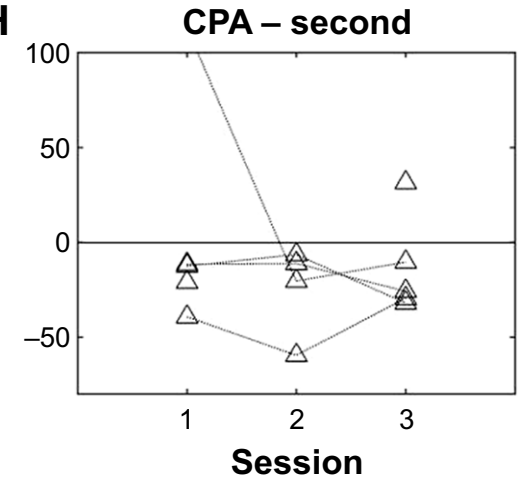

Figure 7 (A) ERD of mean alpha activity as a function of time, from 4 seconds before pressing the button to 2 seconds after button press for three participants: a control participant (CRT; black line), an adult patient with hemiparesis (CPA; red line) and an adolescent with CP (CPY; blue line). Results were obtained for movements with the left hand. (B) The same as in (A) for the right hand. Participant CPA had a hemiparesis of the right side and was unable to perform the test with the right hand. (C-H) Mean ERD values for each experimental session - sessions I, 2 and 3. Each point corresponds to the individual mean ERD value computed within the time window [0.5, I.5] seconds after the button press. Panels (C and D) illustrate results obtained with the best/second hand for individuals in the control group (CTR). The equivalent is represented for individuals in the group of adolescents with CP (CPY) in panels (E and $\mathbf{F})$ and for adult patients (CPA) in panels $(\mathbf{G}$ and $\mathbf{H})$.

Abbreviations: ERD, event-related desynchronization; CP, cerebral palsy. 
consistent changes in performance between sessions. The large changes in performance observed from the first to the second session are not due to body development: not only adult patients showed similar changes, but also, if these changes had been the result of normal development, they would had to be also visible between the second and the third session between which there were also 4 weeks. The most likely explanation for the changes between these sessions is agitation in the first experimental session. This might have affected concentration in the tests, even though all participants had at least one adaptation test run before the recordings. Hence, it seems advisable in this age group and in patients with CP to run a trial test session before any "real" measurements, to reduce any potential influence from excitement for participating.

Excitement might have also affected the recording of alpha brain rhythms, by reducing the number of artifact-free epochs considered in the computation of ERD and, increasing the variability of instant ERD. This contributed to the failure in detecting changes in ERD across sessions and especially with the piano training. Even though clear alpha ERD could be here measured and even though patients with CP show altered sensorimotor brain rhythms, ${ }^{18,33}$ whether piano training or other types of motor training can induce changes in these measures of correlates of motor function remains therefore unclear.

Correlation between regularity in piano keypress during test 1 and performance in the vibration test (Figure 7) is consistent with motor impairments potentially resulting, at least partially, from deficient sensorimotor processing. Perceptual deficits in CP have been reported in previous studies, ${ }^{15,16}$ with alterations being reported also at the level of neuroanatomy, ${ }^{13,32}$ and there is a general consensus that perceptual deficits can be intrinsically associated with motor impairment. ${ }^{14}$

The study was very well received by all participants, and all of them showed interest and continued to receive piano training after the end of the study. This, together with the positive evolution observed in perception tests and with the positive tendency in finger tapping observed for some participants already with such short training, highlights the potential of piano training for personal development in $\mathrm{CP}$ and also the need for further research in this area.

\section{Acknowledgments}

This project was financed by the Frieder-Springer Foundation. The authors would like to thank the support and collaboration of ICP München in this study.

\section{Disclosure}

The authors report no conflicts of interest in this work.

\section{References}

1. Altenmüller E, Marco-Pallares J, Münte TF, Schneider S. Neural reorganization underlies improvement in stroke-induced motor dysfunction by music-supported therapy. Ann N Y Acad Sci. 2009;1169(1): 395-405.

2. Grau-Sánchez J, Amengual JL, Rojo N, et al. Plasticity in the sensorimotor cortex induced by music-supported therapy in stroke patients: a TMS study. Front Hum Neurosci. 2013;7:494.

3. Herholz SC, Zatorre RJ. Musical training as a framework for brain plasticity: behavior, function, and structure. Neuron. 2012;76(3): 486-502.

4. Hund-Georgiadis M, von Cramon DY. Motor-learning-related changes in piano players and non-musicians revealed by functional magneticresonance signals. Exp Brain Res. 1999;125(4):417-425.

5. Gaser C, Schlaug G. Brain structures differ between musicians and non-musicians. J Neurosci. 2003;23(27):9240-9245.

6. Bengtsson SL, Nagy Z, Skare S, Forsman L, Forssberg H, Ullén F. Extensive piano practicing has regionally specific effects on white matter development. Nat Neurosci. 2005;8(9):1148-1150.

7. Hyde KL, Lerch J, Norton A, et al. Musical training shapes structural brain development. J Neurosci. 2009;29(10):3019-3025.

8. Baumann S, Koeneke S, Schmidt CF, Meyer M, Lutz K, Jancke L. A network for audio-motor coordination in skilled pianists and nonmusicians. Brain Res. 2007;1161:65-78.

9. Lappe C, Trainor LJ, Herholz SC, Pantev C. Cortical plasticity induced by short-term multimodal musical rhythm training. PLoS One. 2011; 6(6):e21493

10. Bangert M, Altenmüller EO. Mapping perception to action in piano practice: a longitudinal DC-EEG study. BMC Neurosci. 2003;4(1):26.

11. Schneider S, Schönle P, Altenmüller E, Münte TF. Using musical instruments to improve motor skill recovery following a stroke. J Neurol. 2007;254(10):1339-1346.

12. Rosenbaum P, Paneth N, Leviton A, et al. A report: the definition and classification of cerebral palsy April 2006. Dev Med Child Neurol Suppl. 2007;109:8-14.

13. Hoon AH Jr, Lawrie WJ Jr, Melhem ER, et al. Diffusion tensor imaging of periventricular leukomalacia shows affected sensory cortex white matter pathways. Neurology. 2002;59(5):752-756.

14. Hoon AH Jr, Stashinko EE, Nagae LM, et al. Sensory and motor deficits in children with cerebral palsy born preterm correlate with diffusion tensor imaging abnormalities in thalamocortical pathways. Dev Med Child Neurol. 2009;51(9):697-704.

15. Maitre NL, Barnett ZP, Key AP. Novel assessment of cortical response to somatosensory stimuli in children with hemiparetic cerebral palsy. J Child Neurol. 2012;27(10):1276-1283.

16. Cooper J, Majnemer A, Rosenblatt B, Birnbaum R. The determination of sensory deficits in children with hemiplegic cerebral palsy. J Child Neurol. 1995;10(4):300-309.

17. Nevalainen P, Pihko E, Maenpää H, Valanne L, Nummenmaa L, Lauronen L. Bilateral alterations in somatosensory cortical processing in hemiplegic cerebral palsy. Dev Med Child Neurol. 2012;54(4): 361-367.

18. Pihko E, Nevalainen $P$, Vaalto $S$, et al. Reactivity of sensorimotor oscillations is altered in children with hemiplegic cerebral palsy: a magnetoencephalographic study. Hum Brain Mapp. 2014;35(8):4105-4117.

19. Aisen ML, Kerkovich D, Mast J, et al. Cerebral palsy: clinical care and neurological rehabilitation. Lancet Neurol. 2011;10(9):844-852.

20. Lampe R, Thienel A, Mitternacht J, Blumenstein T, Turova V, AlvesPinto A. Piano training in youths with hand motor impairments after damage to the developing brain. Neuropsychiatr Dis Treat. 2015; 11:1929-1938. 
21. Alves-Pinto A, Turova V, Blumenstein T, Thienel A, Wohlschläger A, Lampe R. fMRI assessment of neuroplasticity in youths with neurodevelopmental-associated motor disorders after piano training. Eur J Paediatr Neurol. 2015;19(1):15-28.

22. Chong HJ, Cho SR, Jeong E, Kim SJ. Finger exercise with keyboard playing in adults with cerebral palsy: a preliminary study. $J$ Exerc Rehabil. 2013;9(4):420-425.

23. Blumenstein T, Turova V, Alves-Pinto A, Lampe R. Sensorimotor piano system for people with disabilities. J Sens. 2016;2016:9825905.

24. Pollok B, Latz D, Krause V, Butz M, Schnitzler A. Changes of motorcortical oscillations associated with motor learning. Neuroscience. 2014;275:47-53.

25. Pfurtscheller G, Lopes da Silva FH. Event-related EEG/MEG synchronization and desynchronization: basic principles. Clin Neurophysiol. 1999;110(11):1842-1857.

26. Pfurtscheller G, Neuper C, Andrew C, Edlinger G. Foot and hand area mu rhythms. Int J Psychophysiol. 1997;26(1-3):121-135.

27. Eliasson AC, Krumlinde-Sundholm L, Röesblad B, et al. The Manual Ability Classification System (MACS) for children with cerebral palsy: scale development and evidence of validity and reliability. Dev Med Child Neurol. 2006;48(7):549-554.
28. Ehrlich S, Alves-Pinto A, Lampe R, Cheng G. A simple and practical sensorimotor EEG device for recording in patients with special needs. Neurotechnix. In Press 2017.

29. Badcock NA, Preece KA, de Wit B, et al. Validation of the Emotiv EPOC EEG system for research quality auditory event-related potentials in children. PeerJ. 2015;3:e907.

30. Delorme A, Makeig S. EEGLAB: an open source toolbox for analysis of single-trial EEG dynamics including independent component analysis. J Neurosci Methods. 2004;134(1):9-21.

31. Kalcher J, Pfurtscheller G. Discrimination between phase-locked and non-phase-locked event-related EEG activity. Electroencephalogr Clin Neurophysiol. 1995;94(5):381-384.

32. Thickbroom GW, Byrnes ML, Archer SA, Nagarajan L, Mastaglia FL. Differences in sensory and motor cortical organization following brain injury early in life. Ann Neurol. 2001;49(3):320-327.

33. Daly I, Faller J, Scherer R, et al. Exploration of the neural correlates of cerebral palsy for sensorimotor BCI control. Front Neuroeng. 2014;7:20.

\section{Publish your work in this journal}

Neuropsychiatric Disease and Treatment is an international, peerreviewed journal of clinical therapeutics and pharmacology focusing on concise rapid reporting of clinical or pre-clinical studies on a range of neuropsychiatric and neurological disorders. This journal is indexed on PubMed Central, the 'PsycINFO' database and CAS, and is the official journal of The International Neuropsychiatric Association (INA). The manuscript management system is completely online and includes a very quick and fair peer-review system, which is all easy to use. Visit http://www.dovepress.com/testimonials.php to read real quotes from published authors. 